\title{
Acetazolamide and chronic hypoxia: effects on haemorheology and pulmonary haemodynamics
}

\author{
Aurélien Pichon*, Philippe Connes", , Patricia Quidu*, Dominique Marchant*, \\ Julien Brunet*, Bernard I. Levy ${ }^{+}$, José Vilar ${ }^{+}$, Innocent Safeukui ${ }^{\S}$, \\ Florence Cymbalista ${ }^{f}$, Maxime Maignan*, Jean-Paul Richalet* and Fabrice Favret ${ }^{\star, * \star}$
}

ABSTRACT: We tested the effect of acetazolamide on blood mechanical properties and pulmonary vascular resistance (PVR) during chronic hypoxia.

Six groups of rats were either treated or not treated with acetazolamide (curative: treated after 10 days of hypoxic exposure; preventive: treated before hypoxic exposure with $40 \mathrm{mg} \cdot \mathrm{kg}^{-1} \cdot \mathrm{day}^{-1}$ ) and either exposed or not exposed to 3 weeks of hypoxia (at altitude $>5,500 \mathrm{~m}$ ). They were then used to assess the role of acetazolamide on pulmonary artery pressure, cardiac output, blood volume, haematological and haemorheological parameters.

Chronic hypoxia increased haematocrit, blood viscosity and PVR, and decreased cardiac output. Acetazolamide treatment in hypoxic rats decreased haematocrit (curative by $-10 \%$ and preventive by $-11 \%$ ), PVR (curative by $-36 \%$ and preventive by $-49 \%$ ) and right ventricular hypertrophy (preventive $-20 \%$ ), and increased cardiac output (curative by $+60 \%$ and preventive by $+115 \%$ ). Blood viscosity was significantly decreased after curative acetazolamide treatment $(-16 \%)$ and was correlated with PVR $(r=0.87, p<0.05)$, suggesting that blood viscosity could influence pulmonary haemodynamics. The fall in pulmonary vascular hindrance (curative by $\mathbf{- 2 7 \%}$ and preventive by $-45 \%$ ) after treatment suggests that acetazolamide could decrease pulmonary vessels remodelling under chronic hypoxia.

The effect of acetazolamide is multifactorial by acting on erythropoiesis, pulmonary circulation, haemorheological properties and cardiac output, and could represent a pertinent treatment of chronic mountain sickness.

KEYWORDS: Acetazolamide, blood volume, chronic hypoxia, haemorheology, pulmonary hypertension

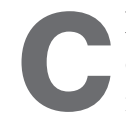
hronic exposure to hypoxia induces polycythaemia and pulmonary hypertension in both humans and rats [1-4]. An excessive erythrocytosis (haemoglobin $(\mathrm{Hb})$ concentration $>21 \mathrm{~g} \cdot \mathrm{dL}^{-1}$ or haematocrit (Hct) concentration $>63 \%$ ) characterises Monge's disease or chronic mountain sickness [5]. Pulmonary hypertension is often associated with chronic mountain sickness [6]. This disease affects $5-15 \%$ of the population living 3,200 $\mathrm{m}$ above sea level.

It has been recently shown that acetazolamide, an inhibitor of carbonic anhydrase, is able to decrease Hct and pulmonary vascular resistance (PVR) in Monge's disease [3, 4]. However, the mechanisms by which acetazolamide could act on PVR are not fully understood. It has been suggested that after a 3-month treatment by acetazolamide the decrease in PVR is due to a decrease in blood viscosity, secondary to the reduction of Hct [4]. According to Poiseuille's law and HOFFMAN [7], pulmonary haemodynamics and PVR may be widely influenced by blood viscosity. However, no data are currently available regarding the effects of acetazolamide on blood viscosity. The changes in PVR observed in chronic hypoxia could be related to several mechanisms, such as pulmonary vascular remodelling $[2,8]$, haemorheological changes [9, 10], hypoxic vasoconstriction [11] or greater nitric oxide scavenging by erythrocytes as a consequence of the rise of Hct [12]. Polycythaemia in various models (mice, rats and guinea pigs) appears to be involved in pulmonary hypertension by increasing blood viscosity [13]. Blood viscosity depends on Hct but also on the ability of
AFFILIATIONS

*Université Paris 13, Sorbonne Paris Cité, Gr-Ex, Laboratoire Réponses cellulaires et fonctionnelles à I'hypoxie EA 2363, ${ }^{f}$ APHP, Hôpital Avicenne, Service d'Hématologie Biologique, Bobigny, and

\# INSERM UMR-S 665, Université Paris Diderot - Paris 7,

${ }^{+}$Cardiovascular Research Center, INSERM U970, Hôpital Européen Georges Pompidou, Université Paris 5 ${ }^{\S}$ Institut Pasteur, Unité d'Immunologie Moléculaire des Parasites, URA CNRS 2581, Paris, and

- UMR Inserm 665, Université des Antilles et de la Guyane, CHU de Pointe à Pitre, Guadeloupe, and **"Mitochondrie, stress oxydant et protection musculaire", EA 3072 , Faculté de Médecine, Université de Strasbourg, Strasbourg, France.

CORRESPONDENCE

A. Pichon

Université Paris 13

Laboratoire "Réponses cellulaires et fonctionnelles à l'hypoxie EA 2363

Bobigny

France

E-mail: aurelion.pichon@orange.fr

Received:

Dec 092011

Accepted after revision: Feb 132012

First published online: April 202012 
red blood cells (RBCs) to deform and aggregate [14]. These latter haemorheological factors may considerably modulate blood flow resistance at the lung level [14-16] and can be influenced by chronic hypoxic exposure [10,17].

The objective of the present study was to improve understanding of the mechanisms involved in the reduction of PVR after acetazolamide treatment under chronic hypoxia conditions with a special emphasis on haemorheological parameters (blood viscosity, aggregation-disaggregation and deformability of RBCs) and pulmonary vascular hindrance, which correspond to the contribution of pulmonary vascular geometry of PVR. We hypothesised that acetazolamide could reduce PVR through a reduction of hypoxic pulmonary vasoconstriction and blood viscosity.

\section{METHODS}

\section{Animals}

All procedures were performed in agreement with the Directive 2010/63/EU of the European Parliament and with the approval of the French "Ministère de l'Agriculture" guidelines (authorisation number A-93-008-01).

Male Wistar rats (Janvier, Le Genest Saint-Isle, France), 8- to 10-weeks old, were divided into six groups: 1) normoxic controls $(n=30) ; 2)$ normoxic curative $(n=24) ; 3)$ normoxic preventive $(\mathrm{n}=32) ; 4)$ hypoxic control $(\mathrm{n}=28) ; 5)$ hypoxic curative $(n=28)$ and 6) hypoxic preventive $(n=32)$. Both preventive groups received acetazolamide 2 days before entering the hypobaric chamber, while curative groups received acetazolamide after 10 days of hypoxic exposure, which has previously been shown to significantly increase pulmonary arterial pressure (PAP) [1]. Rats undergoing chronic hypoxic exposure were housed for 21 days in a hypoxic chamber connected to a gas pump where air was circulated at a pressure of $380 \mathrm{mmHg}(5,500 \mathrm{~m}$ altitude above sea level). Normoxic groups were kept outside the hypobaric chamber at $760 \mathrm{mmHg}$. Treatment by acetazolamide $\left(40 \mathrm{mg} \cdot \mathrm{kg}^{-1} \cdot \mathrm{day}^{-1}\right.$; Sanofi-Aventis, Paris, France) was administered in drinking water and was recorded daily by measuring the amount of acetazolamide drunk per cage during each opening of the hypobaric chamber (30 min per day).

\section{Haemodynamics}

At the end of hypoxic exposure, eight rats from each group were anaesthetised by xylasine-ketamine (intraperitoneal xylasine (Rompum; Bayer, Wuppertal, Germany) $10 \mathrm{mg} \cdot \mathrm{kg}^{-1}$ and ketamine (Clorketam ${ }^{\circledR}$ 1000; Vetoquinol, Lure, France) $\left.50 \mathrm{mg} \cdot \mathrm{kg}^{-1}\right)$. During surgical experiments, the depth of anaesthesia was monitored by the loss of the pedal withdrawal reflex and then by monitoring cardiac frequency $(f c)$ and changes in mean systemic arterial blood pressure. If necessary, an additional dose of the anaesthesia preparation was given intravenously to the animal. All dynamic procedures and biological measurements were completed within $45 \mathrm{~min}$.

The haemodynamic measurements were performed in normoxia to assess chronic effect of acetazolamide and hypoxic exposure according to the method of RABINOVITCH et al. [2]. $f C$, mean systemic arterial blood pressure and PAP were measured by catheterisation. Cardiac output was obtained using thermodilution method.
PVR and systemic vascular resistance (SVR) were estimated by dividing the mean arterial blood pressure (pulmonary and systemic, respectively) by cardiac output. Measurements of the right and left auricular pressures were not performed in rats in the present protocol as the techniques available in the literature are not easy to apply [18] with a high risk of heart failure. Hence, PVR and SVR calculations did not take into account the right and left auricular pressures.

A 300- $\mu \mathrm{L}$ blood sample was removed from femoral artery and analysed to measure arterial oxygen tension $\left(\mathrm{Pa}_{\mathrm{a}} \mathrm{O}_{2}\right)$ and arterial carbon dioxide tension $\left(\mathrm{Pa}, \mathrm{CO}_{2}\right)$. At the end of these experiments, a lethal dose of thiopenthal sodium (pentothal (CEVA Santé Animale, Libourne, France) $100 \mathrm{mg} \cdot \mathrm{kg}^{-1}$ ) was injected and the heart was removed. The right ventricle (RV) and the left ventricle including the septum $(\mathrm{LV}+\mathrm{S})$ were separated, weighed and frozen in liquid nitrogen. Fulton's ratio $(\mathrm{RV} /(\mathrm{LV}+\mathrm{S}))$ was calculated to reflect the right ventricular hypertrophy.

\section{Measurements of blood volume}

An additional experiment was performed on 10 rats for each subgroup to measure blood volume changes under hypoxia and acetazolamide, as compared with normoxic condition. Plasma volume was measured by means of the Evans' blue (Sigma, Gillingham, UK) dye method. Briefly, $100 \mu \mathrm{g}$ of dye in $100 \mu \mathrm{L}$ saline was injected via the jugular venous catheter. The catheter had previously been filled with saline and, after the dye administration, it was flushed with $200 \mu \mathrm{L}$ saline. After 0 , 3,5 and $7 \mathrm{~min}$, saline was removed from the arterial catheter and a blood sample was collected into a heparinised syringe. The blood was centrifuged in order to obtain a plasma sample. The Evans' blue concentration in the plasma sample was determined spectrophotometrically at $610 \mathrm{~nm}$ (Spectrophotometer UV-VIS RS 0223; Jenway 6405; Keison Products, Chelmsford, UK) and compared with a standard curve designed from known amounts of Evans' blue dye and samples of plasma from donor rats. To take into account the progressive leak of Evans' blue from plasma compartment we used a simple two-compartment model to calculate Evans' blue concentration at steady state $\left([E B]_{0}\right)$. Plasma volume was calculated as plasma volume $=\operatorname{IEB} /[\mathrm{EB}]_{0}$, where IEB is the amount of injected Evans' blue $(100 \mu \mathrm{g})$. Then, blood volume and red cell volume (RCV) were calculated as blood volume $=($ plasma volume $\times 100) /(100-H c t)$ and $R C V=$ blood volume-plasma volume.

\section{Haemorheological, haematological and vascular hindrance measurements}

Haemorheological parameters were measured within $3 \mathrm{~h}$ after blood collection in EDTA tubes and in line with the recent international guidelines for haemorheological measurements in 12 rats for each subgroup [19]. Blood viscosity at a shear rate of $36.69 \mathrm{~s}^{-1}$ at $37^{\circ} \mathrm{C}$ and Hct/blood viscosity ratio, which is defined as a haemorheological index of oxygen transport efficiency, were calculated. The RBC elongation indexes were determined at $37^{\circ} \mathrm{C}$ and at seven shear stresses ranging from 0.95 to $30 \mathrm{~Pa}$. The elongation indexes values (data not shown) were plotted as a function of shear stress, and the shear stress required for half-maximal deformation and the predicted maximal elongation indexes were determined by fitting to a Lineweaver-Burk curve [19]. RBC aggregation index and the 
minimal shear rate to prevent $\mathrm{RBC}$ aggregation (disaggregation threshold) were determined at $37^{\circ} \mathrm{C}$ after adjustment of Hct to $40 \%$ [19].

An haematological analyser was used to assess RBCs, white cells and platelets count, mean cell volume, mean corpuscular $\mathrm{Hb}$ concentration $([\mathrm{Hb}])$ and the percentage of reticulocytes.

The pulmonary and systemic vascular hindrances, parameters reflecting the contribution of geometric factors to PVR and SVR, were calculated by dividing mean PVR and mean SVR, respectively, with blood viscosity [20]. A change in vascular hindrance may be interpreted as an effect of acetazolamide and/or hypoxia on pulmonary remodelling or vasoconstriction/vasodilation.

\section{Statistical analysis}

Data are expressed as mean \pm SD. The normality of distribution was assessed by the Kolmogorov-Smirnov test. The effects of environment (normoxia and hypoxia) and drugs (vehicle, acetazolamide curative and acetazolamide preventive) were tested by using an ANOVA with independent groups. Newman-Keuls' test was used for post-hoc test. Unpaired ttest was performed whenever appropriate. Correlations were assessed by using the Pearson test. For exponential regression, the p-value was obtained after logarithmic transformation for linearisation and with the Pearson test. All statistical analyses were performed using the StatSoft statistical software (StatSoft, Inc., Tulsa, OK, USA). A p-value $<0.05$ was considered as a significant difference.

\section{RESULTS}

\section{Heart morphological data}

As expected, chronic exposure to hypoxia induced right ventricular hypertrophy, which was moderated by preventive treatment with acetazolamide (table 1), as assessed by the Fulton's ratio as well as the right ventricular weight/body weight ratio.

\section{Haemodynamics}

PAP increased following chronic hypoxia while acetazolamide has no significant effect (fig. 1a). We observed a main effect of treatment with a lower PAP only in the preventive acetazolamide group compared with the control group, regardless of normoxic or hypoxic exposure.

Cardiac output was not changed by hypoxic exposure although acetazolamide increased cardiac output in hypoxic rats versus hypoxic control rats (fig. 1b). This increase was related to an improvement in stroke volume without any change in $f C$ (table 1).

PVR increased in hypoxic control rats but decreased with acetazolamide treatments. The decrease in PVR was larger in hypoxic preventive than in hypoxic curative rats (fig. 1c). SVR increased in hypoxia and decreased in hypoxic preventive and hypoxic curative rats in comparison with the hypoxic control group (table 1).

\section{Blood volume}

Following hypoxic exposure, RBC volume increased significantly regardless of curative or preventive treatments when compared with the normoxic control group (table 2). Plasma volume decreased significantly after hypoxic exposure in the hypoxic control group when compared with the normoxic control group. No significant difference was found between the normoxic control group and both the hypoxic curative and preventive groups indicating that acetazolamide treatments restored the plasma volume to normoxic values.

\section{Acid-base balance and haematological parameters}

Chronic exposure to hypoxia, as well as acetazolamide treatment, decreased serum bicarbonate, base excess and $\mathrm{pH}$ without difference between curative and preventive treatment, showing that treatment was taken well by rats (table 3). Although measurements were done in normoxia, acclimatised rats showed an increase in $\mathrm{Pa}_{\mathrm{a}} \mathrm{O}_{2}$ that was associated with a decrease in $\mathrm{Pa}_{1} \mathrm{CO}_{2}$, suggesting ventilatory acclimatisation to

TABLE 1 Morphological and cardiovascular parameters observed after 21 days in normoxia or hypoxia in the control, the curative and the preventive groups of rats

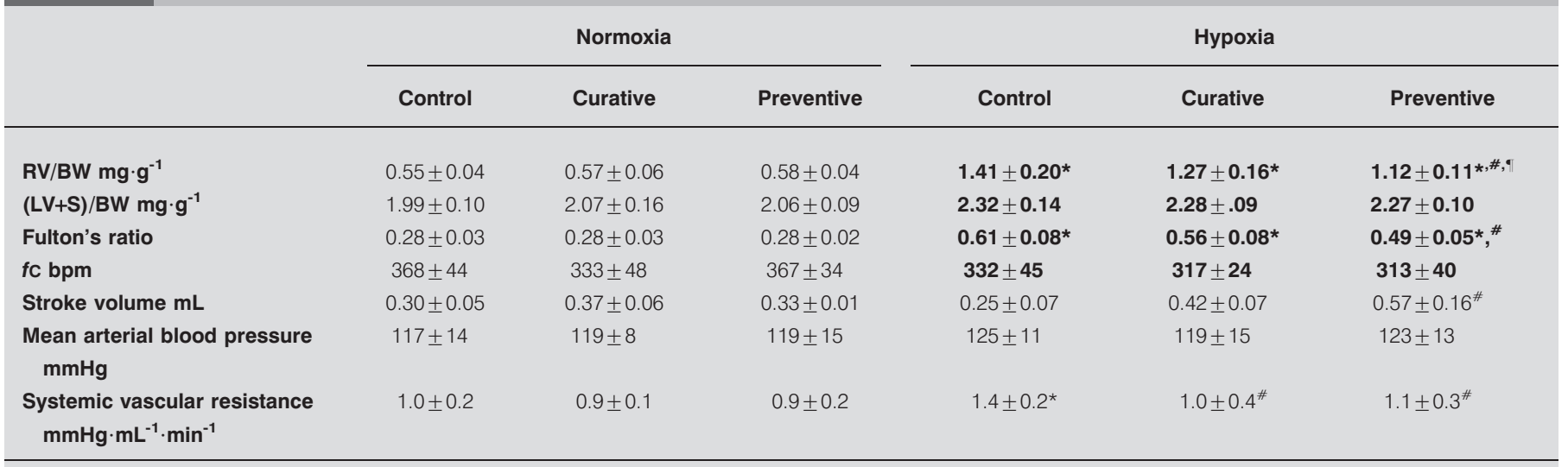

Data are presented as mean \pm SD. RV: right ventricular weight; BW: bodyweight; LV: left ventricular weight; S: septum; fC: cardiac frequency. *: significantly different from normoxia, $\mathrm{p}<0.05$; ${ }^{*}$ : significantly different from the hypoxic control group, $\mathrm{p}<0.05$; ${ }^{\circ}$ : significantly different from the hypoxic curative group, $p<0.05$. Bold indicates main effect of hypoxia, $p<0.05$. 

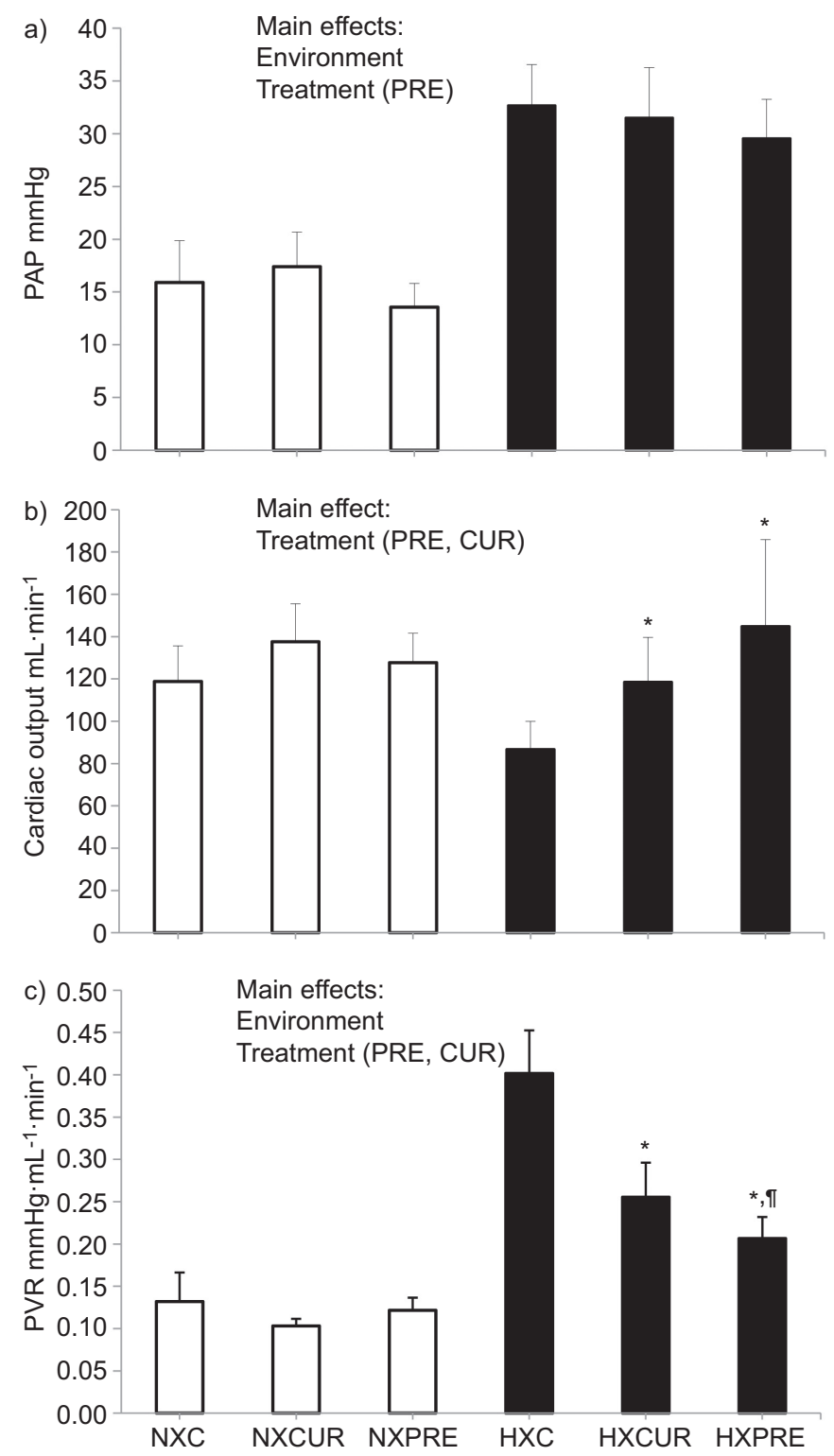

FIGURE 1. Changes in a) pulmonary arterial pressure (PAP), b) cardiac output and c) pulmonary vascular resistance (PVR) after 3 weeks of normoxia (NX) or hypoxia (HX) for the control groups (C), and curative (CUR) and preventive (PRE) acetazolamide treatment groups. *: significantly different from the hypoxic control group, $p<0.05$; ${ }^{\top}$ : significantly different from the hypoxic curative group, $p<0.05$.

hypoxia. There was no significant effect of acetazolamide treatment on the estimated alveolar to arterial oxygen pressure difference $(p=0.57)$ despite opposite tendencies between normoxic and hypoxic groups of rats.

Hypoxia increased Hct, [Hb], RBC count, mean cell volume, percentage of reticulocytes and decreased platelets count in all groups (table 3), while acetazolamide decreased Hct and reticulocytes in both preventive and curative treatment under hypoxia. Although $[\mathrm{Hb}]$ slightly decreased with acetazolamide under hypoxia, the changes did not reach statistical significance ( $-5 \%$ for the curative group and $-7 \%$ for the preventive group). Mean cell volume slightly decreased after the preventive treatment in hypoxia.

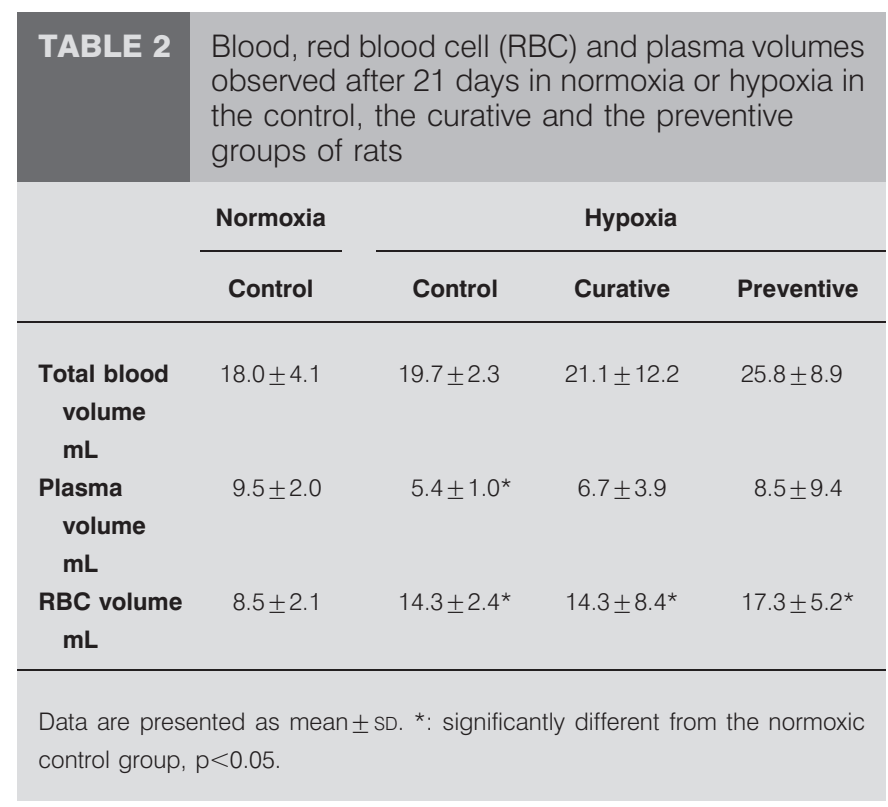

\section{Haemorheological parameters and vascular hindrance}

Haemorheological results are reported in table 4. Whereas hypoxic exposure increased blood viscosity, curative acetazolamide treatment reduced $(-16 \%)$ and preventive acetazolamide treatment tended to decrease it $(-8 \%, p=0.1)$. Neither RBC deformability nor the RBC aggregation index were affected by hypoxia or treatment. The RBC disaggregation threshold decreased with hypoxia but was not affected by acetazolamide treatments. The Hct/viscosity ratio was significantly higher in hypoxic preventive as compared with hypoxic control rats.

For all groups of rats either treated or not with acetazolamide, PVR was significantly related to blood viscosity with an exponential relationship $(p=0.015$; fig. $2 a)$. The association between SVR and blood viscosity was close to be statistically significant ( $p=0.06$; fig. $2 b$ ).

Pulmonary vascular hindrance of hypoxic rats increased in hypoxic control when compared with normoxic control rats (fig. 3a), but decreased with acetazolamide treatments when compared with hypoxic control, with the effect of acetazolamide being more pronounced in the preventive than in the curative group. These findings suggest a direct effect of acetazolamide on pulmonary vasculature (fig. 3a). Despite no difference between normoxic and hypoxic control groups (no effect of chronic hypoxia), the systemic vascular hindrance also decreased in curative and preventive hypoxic groups when compared with the hypoxic control group (fig. 3b). Moreover, when plotting the changes of vascular resistance versus vascular hindrance (fig. 4), acetazolamide decreased both PVR and SVR by acting on pulmonary and systemic vascular hindrance.

\section{DISCUSSION}

Our results show for the first time that the use of acetazolamide after chronic hypoxic exposure, in either preventive or curative treatment, is efficient to decrease 1) Hct, via a restoration of plasma volume; 2) blood viscosity; 3) PVR and SVR; and 4) right ventricular hypertrophy (only after 
TABLE 3 Haematological parameters observed after 21 days in normoxia or hypoxia in the control, the curative and the preventive rats groups

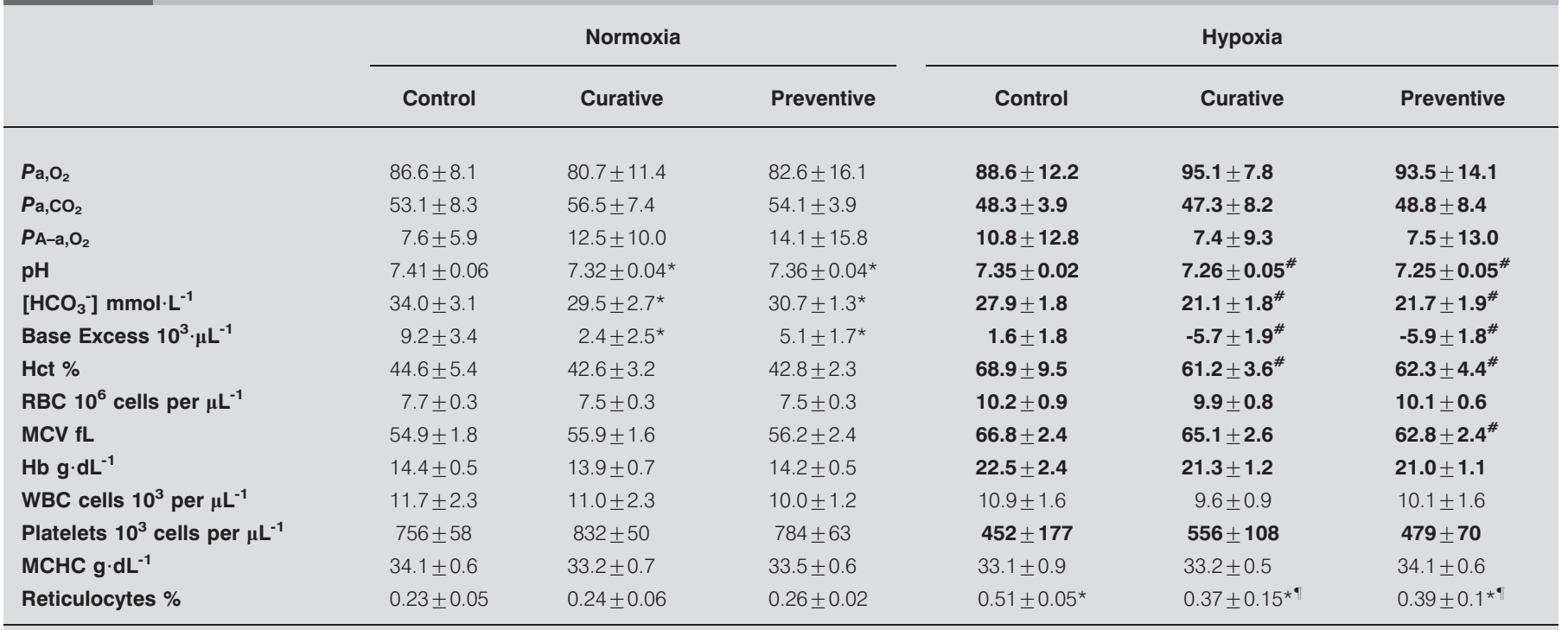

Data are presented as mean $\pm \mathrm{SD} . \mathrm{Pa}, \mathrm{O}_{2}$ : arterial oxygen tension; $\mathrm{Pa}_{2} \mathrm{CO}_{2}$ : arterial carbon dioxide tension; $P \mathrm{~A}-\mathrm{a}, \mathrm{O}_{2}$ : alveolar-arterial oxygen tension difference; $\left[\mathrm{HCO}_{3}{ }^{-}\right.$] blood bicarbonate concentration; Hct: haematocrit; RBC: red blood cell; MCV: mean corpuscular volume; Hb: haemoglobin; WBC: white blood cell; MCHC: mean corpuscular haemoglobin concentration. *: significantly different from the normoxia control group, $\mathrm{p}<0.05$; \#: significantly different from the hypoxic control group $\because$ : significantly different from normoxia, $\mathrm{p}<0.05$. All measurements were performed in normoxia. Bold indicates significant difference from normoxia, main effect, $\mathrm{p}<0.05$

preventive treatment). The relationships between blood viscosity or pulmonary/systemic vascular hindrance and PVR/ SVR suggest that the beneficial effects of acetazolamide on haemodynamics involve haemorheological and pulmonary/ systemic vasculature adaptations.

The observed fall in serum bicarbonate and base excess suggests that the treatment, although given in drinking water, was taken well by rats in normoxia as well as in hypoxia. As water consumption usually decreases at the beginning of hypoxic exposure, acetazolamide treatment started 2 days before hypoxia in the preventive group to obtain an adequate dose of acetazolamide. In curative-treated rats, the treatment began after 10 days of hypoxic exposure, as it has been previously reported that pulmonary hypertension as well as $\mathrm{Hb}$ concentration remain unchanged from day 10 to day 21 [1, 8]. After the first day of acetazolamide treatment, we observed an important increase in water consumption in rats, demonstrating that acetazolamide was also taken well in curative treatment. As previously shown in humans, acetazolamide treatment slightly decreased Hct in both preventive and curative groups [3].

TABLE 4 Haemorheological parameters observed after 21 days in normoxia or hypoxia in the control, the curative and the preventive groups of rats

\begin{tabular}{|c|c|c|c|c|c|c|}
\hline & \multicolumn{3}{|c|}{ Normoxia } & \multicolumn{3}{|c|}{ Hypoxia } \\
\hline & Control & Curative & Preventive & Control & Curative & Preventive \\
\hline Hct/blood viscosity a.u. & $1.32 \pm 0.13$ & $1.27 \pm 0.05$ & $1.28 \pm 0.02$ & $1.21 \pm 0.07$ & $1.35 \pm 0.11$ & $1.39 \pm 0.12^{\#}$ \\
\hline \multicolumn{7}{|l|}{ RBC deformability } \\
\hline Maximal elongation index & $0.61 \pm 0.01$ & $0.60 \pm 0.01$ & $0.61 \pm 0.01$ & $0.61 \pm 0.01$ & $0.61 \pm 0.01$ & $0.61 \pm 0.01$ \\
\hline \multicolumn{7}{|l|}{ RBC aggregation } \\
\hline Aggregation index \% & $37.6 \pm 12.8$ & $37.3 \pm 7.4$ & $51.1 \pm 16.7$ & $43.2 \pm 13.5$ & $40.0 \pm 14.9$ & $44.0 \pm 8.4$ \\
\hline Disaggregation threshold $\mathrm{s}^{-1}$ & $53.7 \pm 12.2$ & $52.1 \pm 11.6$ & $56.4 \pm 10.3$ & $44.2 \pm 4.5$ & $45.0 \pm 5.0$ & $49.2 \pm 6.1$ \\
\hline
\end{tabular}

Data are presented as mean \pm SD. Hct: haemocrit; RBC: red blood cell; a.u.: arbitrary units. ${ }^{\#}$ : significantly different from the hypoxic control group. Bold indicates main effect of hypoxia, $\mathrm{p}<0.05$. 

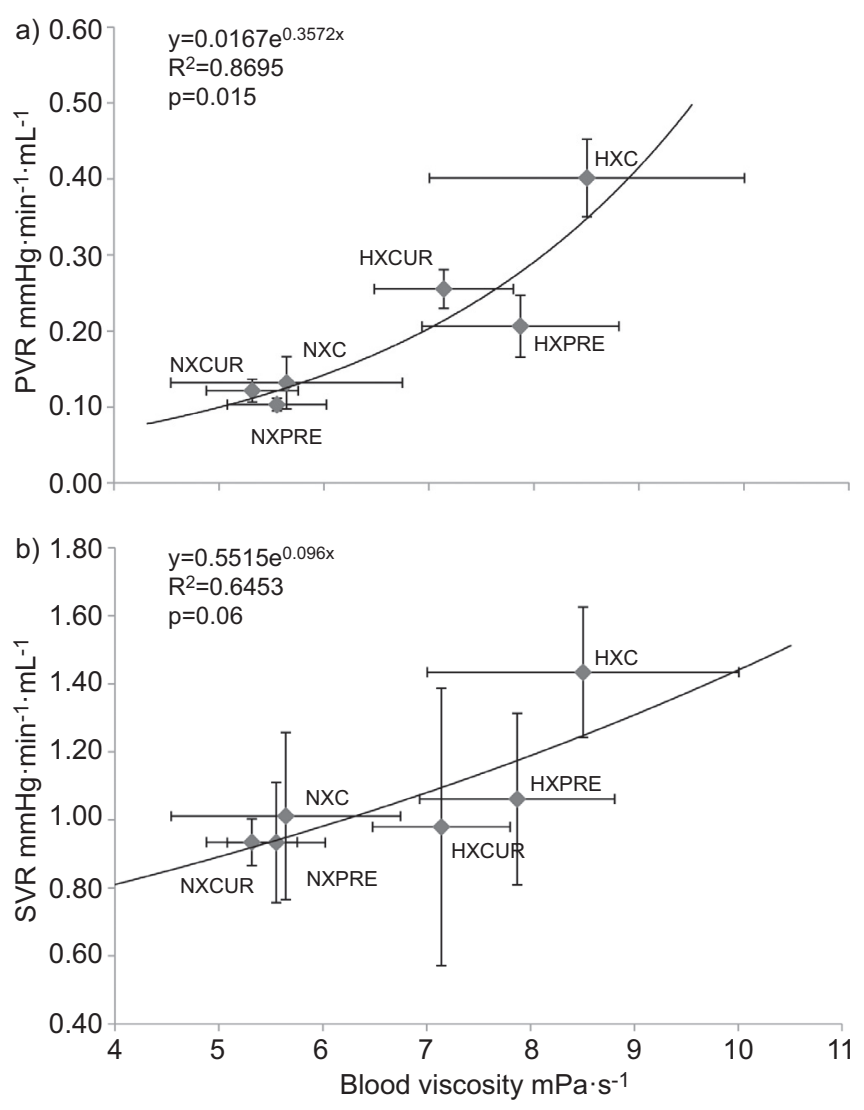

FIGURE 2. Relationship between a) pulmonary vascular resistance (PVR) and b) systemic vascular resistance (SVR) and blood viscosity in rats exposed for 3 weeks to normoxia $(\mathrm{NX})$ or hypoxia $(\mathrm{HX})$ for the control groups $(\mathrm{C})$, and curative (CUR) and preventive (PRE) acetazolamide treatment groups.

As confirmed by our study, exposure to chronic hypoxia results in a fall in plasma volume [21] and an increase in total blood volume secondary to an increase in volume of RBCs [22]. As acetazolamide has diuretic effects, it is expected to further reduce plasma volume in hypoxia, which could offset the decrease in Hct induced by acetazolamide through its stimulatory effects on ventilation and blood oxygenation that may cause a reduction of renal erythropoietin release $[3,4]$. Surprisingly, plasma volume in hypoxic rats treated by acetazolamide was restored to normoxic value and was slightly, although not significantly, increased compared with the hypoxic control rats. It appears that the diuretic effect did not persist in rats chronically treated by acetazolamide and exposed to hypoxia. Therefore, one may suggest that the decrease in Hct in hypoxic rats treated by acetazolamide was not fully explained by the decrease in erythropoiesis (as suggested by the decrease in percentage of reticulocytes), and was also probably the resulting effect of the plasma volume rise, which could be interpreted as a physiological adaptation for maintaining blood volume at the normal value. The hypoxia-induced decrease in plasma volume was probably attributed to the well-known blunting effect of hypoxia on the renin-aldosterone system [21] and other complex mechanisms, such as peripheral arterial chemoreception [23, 24]. The mechanism by which acetazolamide could oppose this blunting effect remains to be established. The increase of ventilation and arterial oxygenation
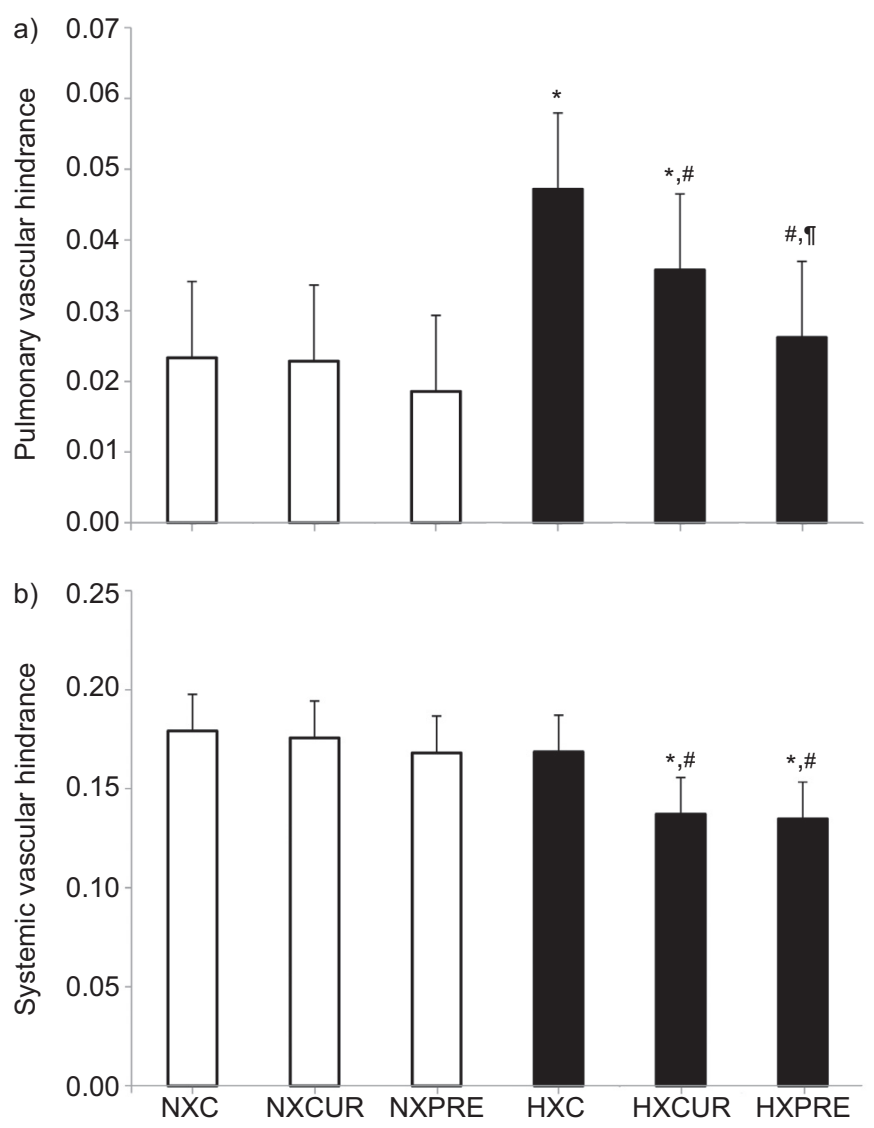

FIGURE 3. a) Pulmonary and b) systemic vascular hindrance calculated in rats exposed for 3 weeks to normoxia (NX) or hypoxia $(H X)$ for the control groups $(C)$, and curative (CUR) and preventive (PRE) acetazolamide treatment groups. *: different from the corresponding normoxic group, $p<0.05$; *: different from hypoxic control group, $p<0.05{ }^{*}$ : different from the hypoxic curative group, $p<0.05$.

under acetazolamide could reduce the hypoxic stimulus for diuresis observed in acute hypoxia [23].

The increase in PVR induced by chronic hypoxia might be due to several factors, such as blood rheological changes [10], hypoxic vasoconstriction or the muscularisation of pulmonary vasculature [2, 8]. Our data do not support a role of aggregation and deformability of RBCs in the increase in PVR under hypoxia. The exact reason for the decrease of the RBC disaggregation threshold under hypoxia is unknown, but one could speculate that the decrease in the platelet count played a role in this finding [25]. Whatever the mechanisms of the change in the $\mathrm{RBC}$ disaggregation threshold under hypoxia, its reduction does not compensate for the increase in Hct and blood viscosity under hypoxia resulting in an increase in PVR.

Although the role of blood viscosity on PVR under hypoxia is not clear [26], RichALET et al. [4] previously suggested that part of the decrease in PVR under acetazolamide treatment could be attributed to a decrease in Hct and blood viscosity [7]. Our results support that the decrease of PVR (in hypoxic conditions) under acetazolamide is related to 1) a decrease in blood viscosity $(-8 \%$ in hypoxic preventive and $-16 \%$ in hypoxic curative groups) and 2 ) a decrease in vascular hindrance $(-45 \%$ 


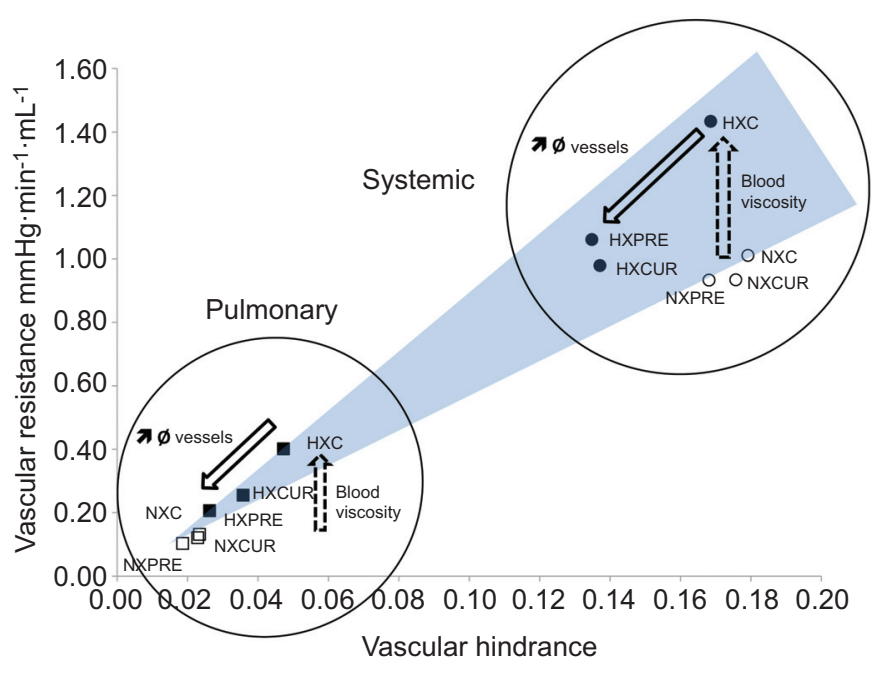

FIGURE 4. Relationship between pulmonary and systemic vascular resistance and vascular hindrance for all groups of rats either treated or not treated with acetazolamide. Dashed arrows represent the only effect of viscosity on systemic vascular resistance due to hypoxia. Solid arrows represent the effect of acetazolamide on vessels diameter. Ø: diameter; NX: normoxia; HX: hypoxia; C: control; CUR: curative; PRE: preventive acetazolamide treatment groups.

in hypoxic preventive and $-35 \%$ in hypoxic curative groups). The significant role played by blood viscosity on PVR is reinforced by the strong correlation found between these two parameters in our study. However, adaptations of vascular geometry in acetazolamide treated rats also need to be considered, as the reduction in vascular hindrance could reflect mainly a reduced pulmonary vascular remodelling and, to a lesser extent, variation in pulmonary vascular tone. Changes in pulmonary vascular tone were probably minor, as our measurements were performed in normoxia, hence limiting the hypoxic pulmonary vasoconstriction phenomenon.

An elegant study conducted in dogs has previously shown that acetazolamide may blunt pulmonary vasoconstriction when animals are submitted to acute severe hypoxia, independently of the decrease in arterial oxygen pressure [27]. Another study observed that acetazolamide could act on pulmonary circulation through a decrease in intracellular calcium in smooth muscle cells during an acute hypoxic challenge [28, 29]. However this effect of acetazolamide on hypoxic pulmonary vasoconstriction is still under discusion in humans, as FAORO et al. [30] failed to reduce it in 10-days acclimatised (3,700$4,700 \mathrm{~m}$ above sea level) sea-level natives acutely treated by acetazolamide, suggesting a possible larger effect of acetazolamide on remodelling as compared with pulmonary vasodilation. In the present study, all measurements of PAP were performed under normoxic conditions. Therefore, the reduction in PVR in hypoxic rats under acetazolamide could be predominantly attributed to a lower pulmonary vascular remodelling and blood hyperviscosity reduction instead of an acute pulmonary vasodilation (figs 3 and 4). These results support the hypothesis that acetazolamide partially prevents and corrects pulmonary remodelling in preventive and curative groups, respectively, resulting in a fall in PVR. The observed decrease in pulmonary vascular hindrance supports this hypothesis. In turn, the decrease in PVR may cause a fall in the right ventricular afterload that might account for the improvement in cardiac output. Further studies are clearly warranted to address the molecular mechanisms by which vascular remodelling could operate under acetazolamide. However, one of the limits in our study is that PVR (as well as SVR) was estimated and not corrected by the left atrial pressure. As blood volume tended to increase in hypoxic acetazolamide rats, it is reasonable to think that the left auricular pressure could have been greater in the acetazolamide group, which could have led to an underestimation of PVR. A second limitation is that all the measurements were performed in normoxia. We were not able to measure the direct effect of chronic acetazolamide treatment on hypoxic pulmonary vasoconstriction and the possible effect of hypocapnia on pulmonary artery pressure. Further studies will have to take into account these limitations.

The lack of statistical difference in blood volume between the hypoxic groups of rats treated or not would suggest that cardiac pre-load is not changed under acetazolamide and, therefore, does not influence cardiac output. However, although not statistically significant, the mean blood volume under preventive treatment was $31 \%$ higher than in the control group after chronic hypoxia. Indeed, it can be speculated that cardiac pre-load was physiologically increased after acetazolamide treatment (more under preventive than under curative conditions), which could have participated in the stroke volume and cardiac output increment through the FrankStarling effect. In addition, this increase in cardiac output observed in hypoxic acetazolamide-treated rats compared with hypoxic controls could be related to the increase in stroke volume secondary to the fall in right ventricular afterload induced by the decrease in PVR. Similarly, sildenafil treatment in patients with secondary pulmonary hypertension increased cardiac output, essentially by reducing right ventricular afterload [3]. Moreover, since acetazolamide increases ventilation and improves alveolar oxygen tension (and $\mathrm{P}_{\mathrm{a}}, \mathrm{O}_{2}$ ), it could reduce the hypoxic pulmonary vasoconstriction and, therefore, limits pulmonary arterial remodelling and the increase in PVR during chronic hypoxia. It is also possible that acetazolamide could improve lung gas exchange efficiency by improved ventilation/perfusion matching. The higher Hct/blood viscosity ratio in hypoxic rats treated with preventive acetazolamide also suggests that the haemorheological efficiency for oxygen transport and tissue oxygenation is effectively improved, limiting the hypoxic stress.

As previously observed, the decrease in PVR directly affects right ventricular weight, moderating right ventricular hypertrophy in preventive-treated rats [2]. The decrease in PVR in curative-treated rats does not result in a significant change in Fulton's ratio. It is reasonable to think that pulmonary remodelling takes several days [8] and, therefore, will affect later the right ventricular afterload in our model of hypoxic exposure (at 21 days). While it has been described that chronic mountain sickness may lead to cardiac failure, our results show that acetazolamide could be efficient on the right heart.

While acetazolamide acts on PVR, our results suggest that it is not a specific effect since this drug decreased SVR too. Although not always observed, several studies have described an increase in SVR in humans and rats exposed to hypoxia 
$[1,31]$. A part of this increase is attributed to a higher Hct in acclimatised rats [31], but other factors are thought to be involved, such as an increase in adrenergic-mediated vasoconstrictor tone and/or vascular remodelling in the systemic circulation [31]. Our results support a role of blood viscosity, as the correlation between blood viscosity and SVR is almost significant. However, hypoxia increased SVR without any increase in vascular hindrance (figs 3 and 4; normoxic control versus hypoxic control groups). Thus, it seems that pulmonary circulation could be more exposed than systemic circulation to remodelling mechanisms under hypoxia. The treatment by acetazolamide had no effect in normoxia, but reduced the systemic vascular hindrance in hypoxic rats leading SVR to return to baseline normoxic values. This statement is in accordance with the results of PICKKERS et al. [32], who showed that acetazolamide could also have some effects on the systemic vasculature.

It should be noted that chronic acetazolamide intake has minor effects on pulmonary vasculature and hypertension compared with either acute high-dose acetazolamide injection [27] or more specific treatments, such as sildenafil or bosentan. Indeed, these two drugs prevent the pulmonary vasoconstrictor response to acute hypoxia and the subsequent development of pulmonary hypertension, right heart hypertrophy and pulmonary arteries remodelling after chronic exposure $[8,33]$.

In conclusion, our present experimental study suggests that both preventive and curative treatments with acetazolamide could be efficient in chronic mountain sickness by moderating PVR through an improvement of the pulmonary vascular geometry. Moreover, acetazolamide increases cardiac output and reduces right ventricular hypertrophy and, therefore, could reduce the long-term risk of cardiac failure. Acetazolamide has an impact on several systems, such as erythropoiesis, blood viscosity, pulmonary circulation, ventilation and cardiac function, and therefore represents an interesting treatment of chronic mountain sickness from a physiopathological point of view.

\section{STATEMENT OF INTEREST}

None declared.

\section{ACKNOWLEDGEMENTS}

We thank M.S. Chambris and S. Varela (University Paris 13) for their work on animal care. We also thank our pharmacist, M. Micoulaud.

\section{REFERENCES}

1 Favret F, Richalet JP, Henderson KK, et al. Myocardial adrenergic and cholinergic receptor function in hypoxia: correlation with $\mathrm{O}_{2}$ transport in exercise. Am J Physiol Regul Integr Comp Physiol 2001; 280: R730-R738.

2 Rabinovitch M, Gamble W, Nadas AS, et al. Rat pulmonary circulation after chronic hypoxia: hemodynamic and structural features. Am J Physiol 1979; 236: H818-H827.

3 Richalet JP, Rivera M, Bouchet P, et al. Acetazolamide: a treatment for chronic mountain sickness. Am J Respir Crit Care Med 2005; 172 : 1427-1433.

4 Richalet JP, Rivera-Ch M, Maignan M, et al. Acetazolamide for Monge's disease: efficiency and tolerance of 6-month treatment. Am J Respir Crit Care Med 2008; 177: 1370-1376.
5 Monge CC, Whittembury J. Chronic mountain sickness. Johns Hopkins Med J 1976; 139: Suppl. 87-89.

6 Antezana AM, Antezana G, Aparicio O, et al. Pulmonary hypertension in high-altitude chronic hypoxia: response to nifedipine. Eur Respir J 1998; 12: 1181-1185.

7 Hoffman JI. Pulmonary vascular resistance and viscosity: the forgotten factor. Pediatr Cardiol 2011; 32: 557-561.

8 Sebkhi A, Strange JW, Phillips SC, et al. Phosphodiesterase type 5 as a target for the treatment of hypoxia-induced pulmonary hypertension. Circulation 2003; 107: 3230-3235.

9 Esteva S, Panisello P, Torrella JR, et al. Blood rheology adjustments in rats after a program of intermittent exposure to hypobaric hypoxia. High Alt Med Biol 2009; 10: 275-281.

10 Palareti G, Coccheri S, Poggi M, et al. Changes in the rheologic properties of blood after a high altitude expedition. Angiology 1984; 35: 451-458.

11 Von Euler US, Liljestrand G. Observations on the pulmonary arterial blood pressure in the cat. Acta Physiol Scand 1946; 12: 301-320.

12 Deem S, Swenson ER, Alberts MK, et al. Red-blood-cell augmentation of hypoxic pulmonary vasoconstriction: hematocrit dependence and the importance of nitric oxide. Am J Respir Crit Care Med 1998; 157: 1181-1186.

13 Hasegawa J, Wagner KF, Karp D, et al. Altered pulmonary vascular reactivity in mice with excessive erythrocytosis. Am J Respir Crit Care Med 2004; 169: 829-835.

14 Baskurt OK, Meiselman HJ. Blood rheology and hemodynamics. Semin Thromb Hemost 2003; 29: 435-450.

15 Doyle MP, Galey WR, Walker BR. Reduced erythrocyte deformability alters pulmonary hemodynamics. J Appl Physiol 1989; 67: 2593-2599.

16 Raj JU, Anderson J. Erythrocyte deformability and lung segmental vascular resistance: effect of hematocrit. J Appl Physiol 1991; 70: 1386-1392.

17 Reinhart WH, Bartsch P. Red cell morphology at high altitude. BMJ Clin Res 1986; 293: 309-310.

18 Casey DB, Badejo AM, Dhaliwal JS, et al. Analysis of responses to the Rho-kinase inhibitor Y-27632 in the pulmonary and systemic vascular bed of the rat. Am J Physiol Heart Circ Physiol 2010; 299: H184-H192.

19 Baskurt OK, Boynard M, Cokelet GC, et al. New guidelines for hemorheological laboratory techniques. Clin Hemorheol Microcirc 2009; 42: 75-97.

20 Chien S, Dellenback RJ, Usami S, et al. Blood volume, hemodynamic, and metabolic changes in hemorrhagic shock in normal and splenectomized dogs. Am J Physiol 1973; 225: 866-879.

21 Richalet JP, Rathat C, Keromes A, et al. Plasma volume, body weight, and acute mountain sickness. Lancet 1983; 1: 525.

22 Ou LC, Sardella GL, Hill NS, et al. Possible role of pulmonary blood volume in chronic hypoxic pulmonary hypertension. J Appl Physiol 1993; 74: 3020-3026.

23 Swenson ER, Duncan TB, Goldberg SV, et al. Diuretic effect of acute hypoxia in humans: relationship to hypoxic ventilatory responsiveness and renal hormones. J Appl Physiol 1995; 78: 377-383.

24 Bouissou P, Richalet JP, Galen FX, et al. Effect of $\beta$-adrenoceptor blockade on renin-aldosterone and $\alpha$-ANF during exercise at altitude. J Appl Physiol 1989; 67: 141-146.

25 Lehmann T, Mairbaurl H, Pleisch B, et al. Platelet count and function at high altitude and in high-altitude pulmonary edema. J Appl Physiol 2006; 100: 690-694.

26 Manier G, Guenard H, Castaing Y, et al. Pulmonary gas exchange in Andean natives with excessive polycythemia-effect of hemodilution. J Appl Physiol 1988; 65: 2107-2117.

27 Hohne C, Krebs MO, Seiferheld M, et al. Acetazolamide prevents hypoxic pulmonary vasoconstriction in conscious dogs. J Appl Physiol 2004; 97: 515-521. 
28 Swenson ER. Carbonic anhydrase inhibitors and hypoxic pulmonary vasoconstriction. Respir Physiol Neurobiol 2006; 151: 209-216.

29 Hohne C, Pickerodt PA, Francis RC, et al. Pulmonary vasodilation by acetazolamide during hypoxia is unrelated to carbonic anhydrase inhibition. Am J Physiol Lung Cell Mol Physiol 2007; 292: L178-L184.

30 Faoro V, Huez S, Giltaire S, et al. Effects of acetazolamide on aerobic exercise capacity and pulmonary hemodynamics at high altitudes. J Appl Physiol 2007; 103: 1161-1165.
31 Gonzalez NC, Erwig LP, Painter CF 3rd, et al. Effect of hematocrit on systemic $\mathrm{O}_{2}$ transport in hypoxic and normoxic exercise in rats. J Appl Physiol 1994; 77: 1341-1348.

32 Pickkers P, Garcha RS, Schachter M, et al. Inhibition of carbonic anhydrase accounts for the direct vascular effects of hydrochlorothiazide. Hypertension 1999; 33: 1043-1048.

33 Chen SJ, Chen YF, Meng QC, et al. Endothelin-receptor antagonist bosentan prevents and reverses hypoxic pulmonary hypertension in rats. J Appl Physiol 1995; 79: 2122-2131. 